\title{
DETERMINAÇÃO DA INCERTEZA DE MEDIÇÃO NOS ENSAIOS DE COMPRESSÃO PARALELA ÀS FIBRAS ${ }^{1}$
}

\author{
Edgar Vladimiro Mantilla Carrasco², Eliene Pires Carvalho² e Ana Lúcia Crespo Oliveira²
}

\begin{abstract}
RESUMO - O objetivo deste trabalho foi apresentar uma metodologia para o cálculo da incerteza de medição do resultado do ensaio de resistência à compressão paralela às fibras. Pretendeu-se reunir subsídios que justifiquem a adoção do Procedimento de Cálculo de Incerteza de Medição como exigência normativa e parte integrante do relatório de ensaios de caracterização de madeiras. A motivação para a apresentação dessa proposta surgiu devido à dificuldade observada no atendimento a alguns requisitos técnicos da norma ABNT 2005 - NBR ISO/ IEC 17025, em especial o requisito 5.9 sobre a “Garantia da Qualidade de Resultados de Ensaio e Calibração”. A metodologia proposta consolida os procedimentos necessários para a obtenção da incerteza de medida individual da tensão de ruptura e o resultado da incerteza da média das tensões de ruptura. Essa metodologia atende aos requisitos de um Sistema de Gestão da Qualidade. Os valores de incerteza obtidos dos resultados individuais da tensão de ruptura foram pouco significativos, indicando elevada qualidade dos equipamentos e boa calibração dos mesmos. Já a incerteza de medição da média da tensão de ruptura foi considerável, indicando a importância de sua consideração na segurança das estruturas de madeira.
\end{abstract}

Palavras-chave: Eucalyptus cloeziana, ensaio de compressão e incerteza de medição.

\section{UNCERTAINTY CALCULATION IN THE COMPRESSION PARALLEL TO GRAIN TESTS}

\begin{abstract}
The purpose of this paper is to present a methodology to determine the uncertainty calculation in the compression parallel to grain strength test. Subsidies were collected to justify the application of the Procedure for Uncertainty Calculation as a normative requirement and integrant part of the report on wood characterization testing. The motivation to the presentation of this proposal is due to the existing difficulty to meet some technical requirements of standard ABNT 2005 - NBR ISO/IEC 17025, especially requirement 5.9 "Quality guarantee of the calibration and testing results". The methodology proposed consolidates all the necessary procedures to obtain the uncertainty calculation for the individual ultimate stress and the mean ultimate stress uncertainty results. This methodology meets the requirements of the Quality Guarantee System. The uncertainty values obtained from the individual results of the ultimate stress were little significant, indicating a high quality of the equipments and their good calibration. On the other hand, determination of the uncertainty of the mean ultimate stress was very significant, indicating its importance to improve the safety of wood engineering.
\end{abstract}

Keywords: Eucalyptus cloeziana, compression testing and uncertainty calculation.

\footnotetext{
${ }^{1}$ Recebido em 20.10.2006 e aceito para publicação em 18.12.2007.

${ }^{2}$ Departamento de Engenharia de Estruturas da Escola de Engenharia, Universidade Federal de Minas Gerais (UFMG), Belo Horizonte-MG. E-mail :<mantilla@dees.ufmg.br>; <eliene@dees.ufmg.br>e <lucia@dees.ufmg.br>.
} 


\section{INTRODUÇÃO}

A Norma ABNT 2005 - NBR ISO/IEC 17025 foi desenvolvida especificamente para laboratórios de ensaios e calibração que desejam implantar um sistema da qualidade. Estabelece mecanismos que visam à confiabilidade e a credibilidade dos ensaios. O cumprimento dos requisitos estabelecidos por essa norma atestam a competência técnica do laboratório na realização de ensaios.

Observa-se que o mercado nacional e, principalmente, o mercado externo estão se tornando bastante exigentes quanto à qualidade dos produtos ofertados ao consumidor, impondo critérios para aceitação de produtos que atendam a requisitos especificados em normas e regulamentos técnicos. Devido à importância estratégica do processo de certificação de produtos, a Divisão de Credenciamento de Laboratórios - DICLA do INMETRO, através da NITDICLA-033 (2005), estabelece a política para que os laboratórios de ensaio acreditados e aqueles postulantes à acreditação implementem a estimativa de incerteza para os ensaios acreditados, requisito obrigatório desde dezembro de 2005, em atendimento ao item 5.9, visando à "Garantia da Qualidade de Resultados de Ensaio e Calibração” da ABNT 2005 - NBR ISO/IEC 17025.

Os ensaios para a determinação da resistência na compressão paralela às fibras são realizados no Brasil segundo a ABNT (1997) - NBR 7190, conforme procedimentos disponibilizados no Anexo B "Determinação das propriedades das madeiras para projeto de estruturas” e, até o momento, não exige um rigor na apresentação dos resultados de ensaio levando em consideração o cálculo da incerteza de medição, como pode ser visto em Serpa, et al. (2003).

No Brasil, verifica-se grande lacuna na realização de ensaios adotando sistemas da qualidade, conforme avaliação de Morel (2004), para o qual é reconhecidamente difícil de se estimar a incerteza de ensaios que utilizam organismos, como espécies animais e vegetais.

Lobão et al. (2004) enfatizaram que são realizados vários estudos na área específica da madeira, com o objetivo de identificar os fatores que afetam as propriedades físico-mecânicas desse material orgânico, sejam eles inerentes à própria madeira ou, também, a fatores ambientais no qual a mesma se desenvolve. A madeira é um material heterogêneo com variações nas suas composições químicas, físicas e anatômicas, que ocorrem entre espécies e dentro da mesma espécie. Essas variações costumam ocorrer devido à idade, posição do elemento analisado na árvore e fatores genéticos, ambientais etc.

Na análise dos fatores que afetam a medição de um material, deve-se considerar que o comportamento dos organismos (como as espécies vegetais) é altamente dependente das condições ambientais do laboratório (MOREL, 2004), ou seja, das grandezas de influência que, segundo o INMETRO (2005), não são o mensurando, porém afetam o resultado da medição deste.

A análise de processos de medição, visando à confiabilidade e exatidão de atividades para atendimento das normas ABNT (2005) - NBR ISO/IEC 17025 e ISO/ TS 14253-1 (1998), motivou os pesquisadores Oliveira e Santos (2004) a apresentarem uma metodologia genérica, cuja aplicação permitiria desenvolver o senso de análise crítica no pessoal técnico envolvido, assim como possibilitar a geração de documento técnico orientativo para outros laboratórios metrológicos. A realização de uma análise crítica formal nos processos de medição possibilitaria a detecção de falhas, visando à implementação das devidas alterações, com o objetivo de promover melhorias contínuas.

\section{MATERIAL E MÉTODOS}

Os dados experimentais apresentados neste trabalho foram retirados do estudo realizado em Mantilla (2006). Sendo a madeira utilizada a da espécie Eucalyptus cloeziana. Foram 62 árvores, e de cada uma retiraramse quatro toretes de $100 \mathrm{~cm}$, objetivando à obtenção do maior número possível de corpos-de-prova válidos. Os toretes ficaram acondicionados em temperatura ambiente até o início dos ensaios. A partir desses toretes, foram preparados todos os corpos-de-prova para os ensaios de caracterização do lote. Para os ensaios de resistência, foram utilizados os seguintes equipamentos: máquina universal de ensaios, marca EMIC; paquímetro digital, marca Mitutoyo; e mutiplexador, marca Mitutoyo.

\section{RESULTADOS E DISCUSSÃO}

\subsection{Resultados do ensaio de compressão paralela às fibras}

Foram ensaiados 248 corpos-de-prova completamente saturados de 62 árvores. Para este trabalho, utilizaram-se apenas 12 corpos-de-prova, 
uma vez que o objetivo era apresentar a metodologia de cálculo da incerteza. No Quadro 1, apresentamse os valores obtidos no ensaio de compressão paralela às fibras.

\subsection{Incerteza de medição nos ensaios de compressão paralela às fibras}

A incerteza de medição compreende, em geral, muitos componentes, alguns dos quais podem ser caracterizados por desvios-padrão experimentais. Os outros componentes, que também podem ser caracterizados por desvios-padrão, são avaliados por meio da distribuição de probabilidades assumidas, baseadas na experiência ou em outras informações. Em regra, consideram-se as seguintes etapas para a estimativa da incerteza de medição: definição do mensurando, das grandezas de entrada e das fontes de incerteza; determinação dos coeficientes de sensibilidade e dos componentes de incerteza; determinação da incertezapadrão combinada; determinação dos graus de liberdade efetivos; e determinação do coeficiente de abrangência e da incerteza expandida. Cada uma dessas etapas será detalhada nos tópicos subseqüentes.

\subsubsection{Definição do mensurando, grandezas de entrada e fontes de incerteza}

O mensurando é a grandeza específica que está sendo submetida à medição. No ensaio em questão, ele é a resistência à compressão paralela às fibras $\left(\mathrm{f}_{\mathrm{c} 0}\right)$ e está relacionado à força de ruptura $(\mathrm{F})$ e à área da seção transversal do corpo-de-prova (A), isto é, $\mathrm{f}_{\mathrm{c} 0}=\mathrm{F} / \mathrm{A}$.
A área, por sua vez, é função das dimensões da seção transversal $\left(D_{1}\right.$ e $\left.D_{2}\right)$; assim, $A=D_{1} \times D_{2}$. Considerando que o mensurando ( $f_{c 0}$ ) está relacionado a várias grandezas de entrada, denominou-se a resistência à compressão $\left(\mathrm{f}_{\mathrm{c} 0}\right)$ de mensurando principal e as demais grandezas de entrada, mensurandos secundários. Eles estão correlacionados no diagrama de causa e efeito apresentado na Figura 1.

Uma vez definidos o mensurando principal e as grandezas de entrada, as fontes de incerteza foram relacionadas no diagrama de causa e efeito, mostrado na Figura 2.

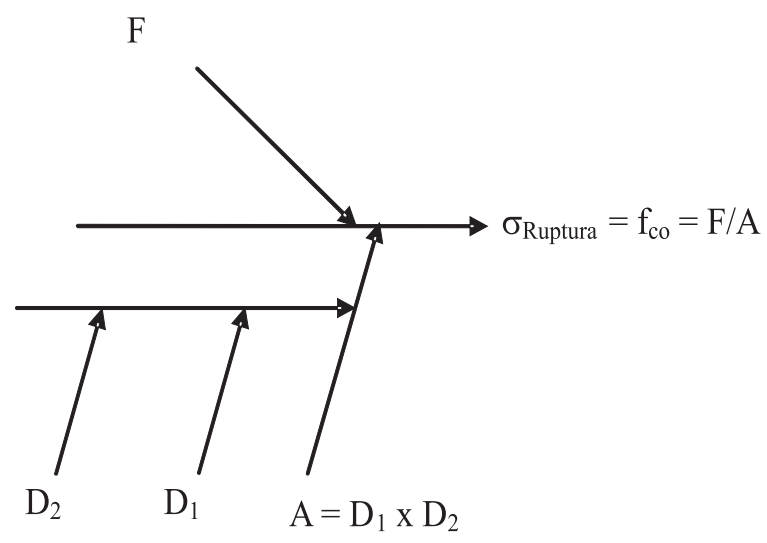

Figura 1 - Diagrama de causa e efeito, mensurando principal e grandezas de entrada.

Figure 1 - Cause and effect diagram - main measure and input data.

Quadro 1 - Resultados dos ensaios de compressão paralela às fibras Table 1 - Results of the compression parallel to grain tests

\begin{tabular}{|c|c|c|c|c|c|}
\hline $\mathrm{CP}$ & Dimensão 1 (mm) & Dimensão 2 (mm) & Área $\left(\mathrm{mm}^{2}\right)$ & Força $(\mathrm{N})$ & $\mathrm{f}_{\mathrm{c} 0}(\mathrm{MPa})$ \\
\hline 1 & 49,69 & 50,42 & 2505,37 & 161975 & 64,65 \\
\hline 2 & 50,30 & 49,91 & 2510,47 & 158510 & 63,14 \\
\hline 3 & 49,82 & 49,92 & 2487,01 & 149642 & 60,17 \\
\hline 4 & 50,80 & 50,51 & 2565,91 & 155769 & 60,71 \\
\hline 5 & 50,67 & 50,43 & 2555,29 & 159812 & 62,54 \\
\hline 6 & 50,17 & 50,32 & 2524,55 & 151521 & 60,02 \\
\hline 7 & 49,15 & 49,87 & 2451,11 & 152431 & 62,19 \\
\hline 8 & 50,29 & 49,65 & 2496,89 & 151746 & 60,77 \\
\hline 9 & 50,61 & 50,32 & 2546,69 & 157805 & 61,96 \\
\hline 10 & 50,45 & 50,30 & 2537,63 & 160291 & 63,17 \\
\hline 11 & 50,66 & 50,24 & 2545,16 & 160898 & 63,22 \\
\hline 12 & 50,60 & 50,60 & 2560,36 & 158441 & 61,88 \\
\hline Média & 50,27 & 50,21 & 2523,87 & 156570 & 62,04 \\
\hline Dês. Pad. & 0,49 & 0,30 & 34,58 & 4217 & 1,41 \\
\hline
\end{tabular}




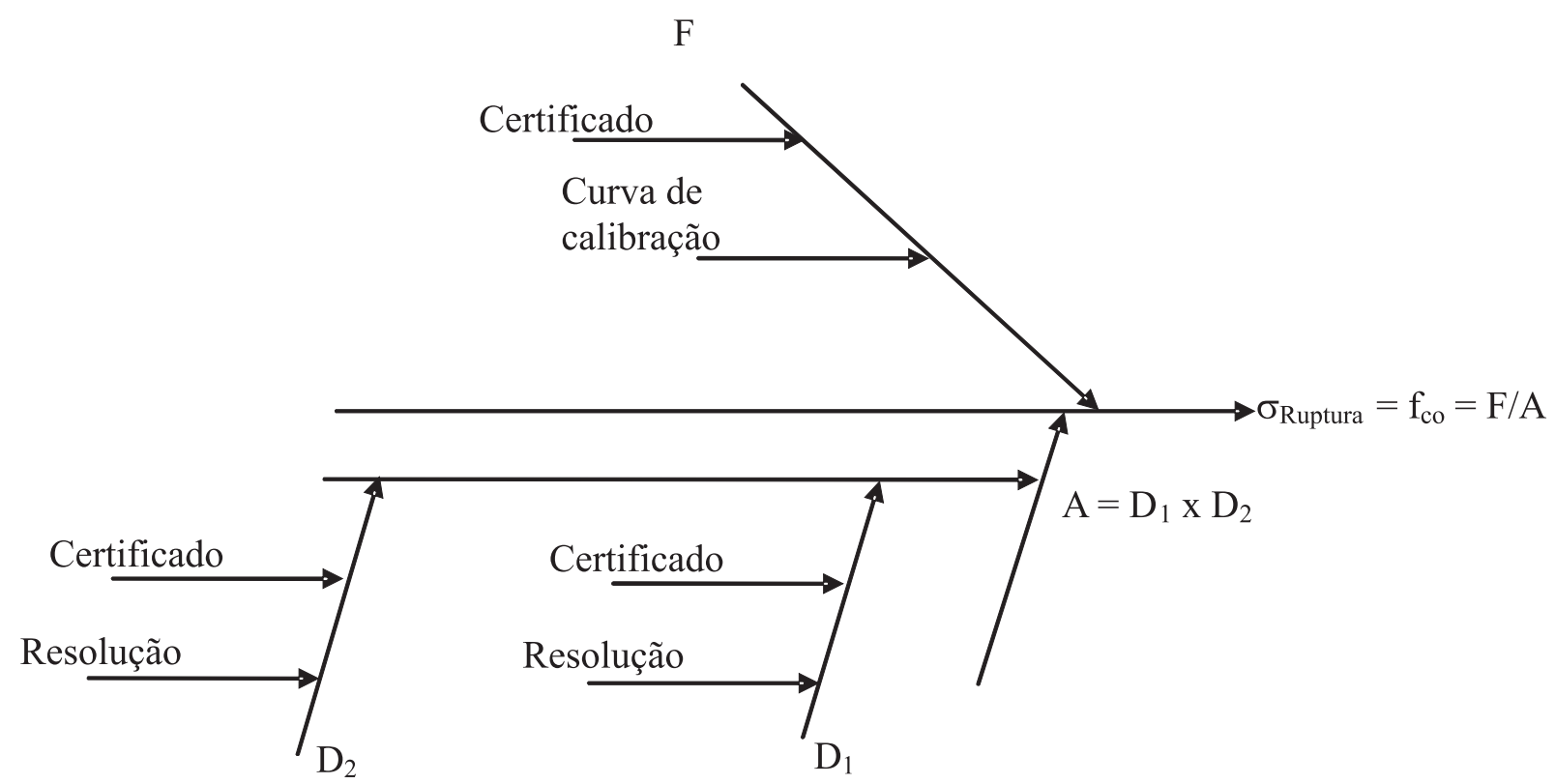

Figura 2 - Mensurando principal, grandezas de entrada e fontes de incerteza.

Figure 2-Main measure, input data and uncertainty sources.

Segundo o Gum (1998), as fontes de incerteza poderão ser do tipo A ou B, conforme sua origem. Incertezas do tipo A são relacionadas à repetitividade do ensaio, ou seja, quando são feitas várias medições para a determinação do valor de uma grandeza, de forma a originar uma média e um desvio-padrão. Incertezas do tipo B podem ser relacionadas à resolução do instrumento, ao seu certificado de calibração, a constantes físicas e a outras estimativas relevantes ao processo de medição.

Neste trabalho, as fontes de incerteza relacionadas à curva de calibração foram consideradas do tipo A e as demais fontes, do tipo B. Elas foram calculadas separadamente e, depois, correlacionadas para determinação da incerteza do mensurando principal $\left(\mathrm{f}_{\mathrm{c} 0}\right)$.

\subsubsection{Cálculo da incerteza}

O cálculo da incerteza do resultado de ensaio, em cada corpo-de-prova, foi iniciado pela incerteza relacionada aos valores de suas dimensões. Em seguida, calculou-se a incerteza relativa à área, à força de ruptura e, finalmente, à tensão de ruptura.

Incerteza das dimensões $D_{1}$ e $D_{2}$ do corpo-de-prova. Observa-se, na Figura 2, que as fontes de incerteza de $\mathrm{D}_{1}$ são: incerteza devida à resolução do equipamento e incerteza declarada no certificado de calibração. A incerteza declarada no certificado de calibração está relacionada a uma probabilidade de ocorrência, através do fator de abrangência k (divisor), considerando-se a distribuição normal. Esse valor de incerteza foi transformado em uma incerteza-padrão, relacionada à probabilidade de ocorrência do valor de um desvio-padrão, utilizandose a equação 1 .

$u_{\text {certificado }}=\frac{U_{(\text {declarada })}}{k}$

em que:

$u=$ incerteza-padrão relacionada a uma fonte de incerteza;

$U=$ incerteza combinada declarada no certificado de calibração, e

$k=$ coeficiente de abrangência declarado no certificado de calibração.

Para a determinação da incerteza relacionada à resolução do paquímetro, admitiu-se uma distribuição de probabilidade de ocorrência tipo triangular. Aincertezapadrão foi calculada utilizando-se a equação 2 . 


$$
u\left(x_{i}\right)=\frac{c}{\sqrt{6}}
$$

em que:

$$
c=\text { resolução do paquímetro. }
$$

A incerteza combinada $\left(u_{c}\right)$ das dimensões $\mathrm{D}_{1} \mathrm{e}$ $\mathrm{D}_{2}$ foi calculada segundo a equação 3 , combinandose as incertezas-padrão já determinadas.

$$
u_{c}^{2}(y)=\sum_{i=1}^{n} u^{2}\left(x_{i}\right)
$$

Incerteza da área. Observa-se que, para o cálculo da incerteza da área, em $\mathrm{mm}^{2}$, é preciso combinar as incertezas de $\mathrm{D}_{1}$ e $\mathrm{D}_{2}$, em mm. Existe, portanto, a necessidade de ponderar os valores de incerteza das grandezas de entrada, além de compatibilizar as unidades de medida. Essa compatibilização foi feita através dos coeficientes de sensibilidade $\left(\mathrm{C}_{\mathrm{i}}\right)$, calculados utilizandose a equação 4 :

$y=f\left(x_{i}\right) e C_{i}=\frac{\partial f}{\partial x_{i}}$

em que:

$$
\begin{aligned}
& y=\text { mensurando; e } \\
& \mathrm{x}_{\mathrm{i}}=\text { grandezas de entrada do mensurando. }
\end{aligned}
$$

A incerteza combinada $\left(u_{c}\right)$ da área foi calculada segundo a equação 5, combinando-se as incertezaspadrão já determinadas.

$$
u_{c}^{2}(y)=\sum_{i=1}^{n}\left[\frac{\partial f}{\partial x_{i}}\right] u^{2}\left(x_{i}\right)
$$

Incerteza da força. De acordo com a Figura 2, as fontes de incerteza da força são: incerteza declarada no certificado de calibração e incerteza devida à curva de calibração. As incertezas declaradas no certificado de calibração da célula de carga da máquina universal, para os pontos calibrados, estão relacionadas a uma probabilidade de ocorrência, através do fator de abrangência k, considerando-se a distribuição normal. Esses valores de incerteza foram transformados em incertezas-padrão, relacionadas à probabilidade de ocorrência para o valor de um desvio-padrão, utilizandose a equação 1. A Figura 3 ilustra a relação entre os pontos calibrados da célula de carga e seus correspondentes valores de incerteza-padrão. Utilizandose a equação obtida por meio da regressão linear, apresentada na Figura 3, foram determinadas as incertezas dos valores de força medidos no ensaio. A incerteza devida à curva de calibração, mostrada na Figura 4, refere-se aos parâmetros da curva de regressão linear, obtida a partir da relação entre os valores dos padrões de calibração (referência) e os valores indicados na máquina universal.

Por exemplo, no ensaio do CP1 o valor indicado pela máquina foi de 161975 N. Essa força corresponde, segundo a curva de calibração, a um valor de referência igual a $162247 \mathrm{~N}$, considerado como valor verdadeiro convencional (VVC). Ao adotarem os valores verdadeiros convencionais das medidas, deve-se considerar uma incerteza relacionada aos parâmetros da curva de calibração que os originaram. Nesse caso, para o cálculo da incerteza relacionada aos parâmetros da curva de regressão linear foram utilizadas as equações 6 a 9 .

$F_{\text {ref }}=a+b F_{\text {ind }}$ $u_{a}=\frac{s}{\sum \sqrt{\left(F_{\text {ind }}-\bar{F}_{\text {ind }}\right)^{2}}}$ (incerteza associada ao

$s^{2}=\frac{\sum \Delta^{2}}{n-2} ; \bar{F}_{\text {ind }}=\frac{F_{\text {ind }}}{n}$

$u_{b}=s \sqrt{\frac{\sum F_{\text {ind }}{ }^{2}}{n \sum\left(F_{\text {ind }}-\overline{F_{\text {ind }}}\right)^{2}}}$ (incerteza associada ao parâmetro b)

em que:

$\Delta=$ diferença entre a força de referência calculada, segundo a curva de regressão, e a força de referência experimental;

$$
\begin{aligned}
& \mathrm{F}_{\text {ref }}=\text { força de referência; } \\
& \mathrm{F}_{\text {ind }}=\text { força indicada; } \\
& \mathrm{n}=\text { número de pontos da curva; e } \\
& \mathrm{s}^{2}=\text { medida aproximada da variância média, quando }
\end{aligned}
$$
é utilizada a equação de regressão para prever a força de referência correspondente a dado valor da força indicada.

R. Árvore, Viçosa-MG, v.32, n.1, p.119-127, 2008 


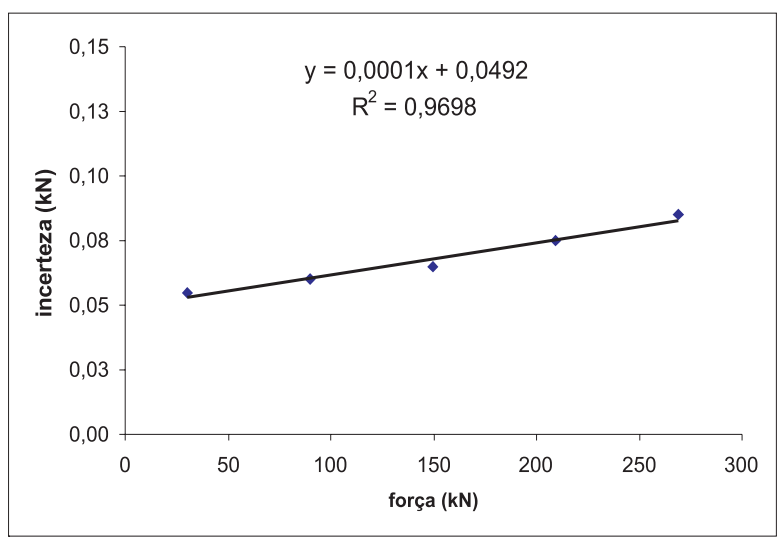

Figura 3 - Relação entre a incerteza-padrão declarada no certificado de calibração e os pontos calibrados.

Figure 3-Relation between the standard uncertainty declared in the calibration certificate and the calibration points.

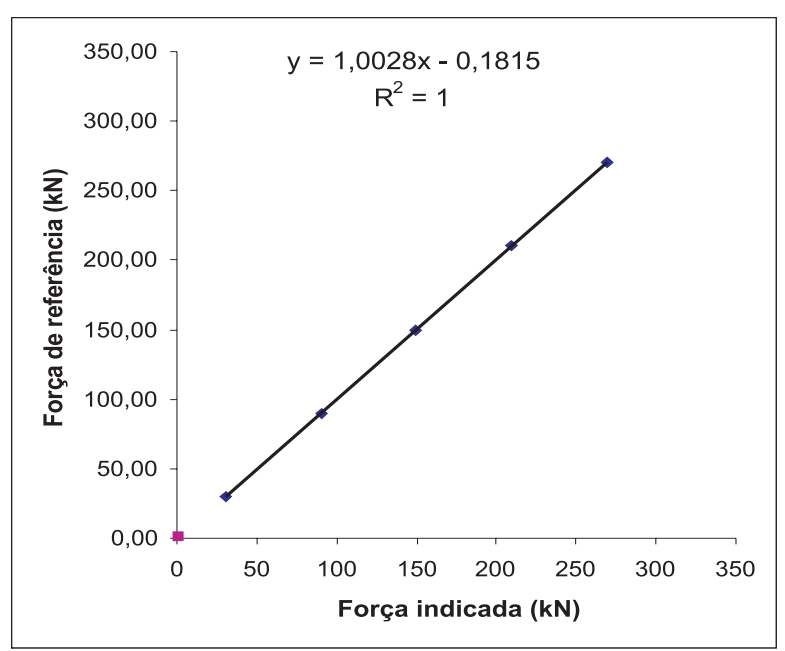

Figura 4 - Curva de calibração da célula de carga utilizada na máquina universal.

Figure 4-Load cell calibration curve used in the Universal Testing Machine.

A incerteza combinada $\left(u_{c}\right)$ entre as incertezas dos parâmetros a e $b$ foi calculada segundo as equações 4,10 e 11 e a incerteza combinada $\left(u_{c}\right)$ da força, determinada utilizando-se a equação 3 , considerando as incertezas-padrão calculadas anteriormente.

$$
\begin{aligned}
& u_{c}^{2}(y)=\sum_{i=1}^{n}\left[\frac{\partial f}{\partial x_{i}}\right] u^{2}\left(x_{i}\right)+2 \sum_{i=1}^{n-1} \sum_{j=i+1}^{n} c_{i} c_{j} u\left(x_{i}\right) \\
& u\left(x_{j}\right)_{a, b}\left(x_{i}, x_{j}\right)
\end{aligned}
$$

$r_{a, b}=-\frac{\sum F_{\text {ind }}}{\sqrt{n \sum F_{\text {ind }}{ }^{2}}}$

em que:

$r_{a, b}=$ coeficiente de correlação entre a e b.

Incerteza da resistência à compressão paralela às fibras $\left(\mathbf{f}_{\mathbf{c} 0}\right)$. Para o cálculo da incerteza da tensão ( $\left.\mathrm{f}_{\mathrm{c} 0}\right)$, em $\mathrm{MPa}$, deve-se combinar as incertezas da força em $\mathrm{N}$ com a incerteza da área em $\mathrm{mm}^{2}$. Existe, da mesma forma que foi feito para a incerteza da área, a necessidade de ponderar as grandezas de entrada para o mensurando, além de compatibilizar as unidades de medida. Essa compatibilização foi feita através dos coeficientes de sensibilidade $\left(\mathrm{C}_{\mathrm{i}}\right)$, calculados utilizandose a equação 4 . A incerteza combinada $\left(u_{c}\right)$ da resistência à compressão paralela às fibras $\left(\mathrm{f}_{\mathrm{c} 0}\right)$ foi determinada adotando-se a equação 3 e considerando as incertezaspadrão da área e da força de ruptura. Ressalta-se que a incerteza calculada para a tensão de ruptura até esse passo é denominada incerteza-padrão e está relacionada à probabilidade de ocorrência para o valor de um desviopadrão. Para o cálculo da incerteza expandida, devem ser calculados os graus de liberdade efetivos e, a partir de uma probabilidade de ocorrência escolhida, determinar um fator de abrangência que será multiplicado pela incerteza-padrão. Os graus de liberdade efetivos, neste trabalho, foram calculados utilizando-se a equação 12 .

$$
v_{\text {eff }}=\frac{u_{c}^{4}(y)}{\sum \frac{\left(C_{i} \times u\left(x_{i}\right)\right)^{4}}{v_{i}}}
$$

em que:

$v_{i}=$ graus de liberdade efetivos associados às incertezas combinadas das grandezas de entrada $\mathrm{u}_{\mathrm{i}}(\mathrm{y})$;

$\mathrm{u}_{\mathrm{c}}=$ incerteza combinada do mensurando;

$\mathrm{u}_{(\mathrm{xi})}=$ incerteza combinada das grandezas de entrada; e

$\mathrm{C}_{\mathrm{i}}=$ coeficiente de sensibilidade, calculado de acordo com a equação 6 .

O valor de $v_{\mathrm{i}}$, para o caso das incertezas do tipo A e para n pontos calibrados, é $v_{\mathrm{i}}=\mathrm{n}-2$ e para as incertezas do tipo B, $v_{\mathrm{i}} \rightarrow 8$.

A incerteza expandida foi determinada utilizandose a equação 13. O fator de abrangência $(\boldsymbol{K})$ foi 
estabelecido a partir da distribuição t de Student, com $v_{\text {eff }}$ (graus de liberdade), para um nível de 95\% de confiança.

$$
U=K \times u_{c}
$$

A planilha utilizada para o cálculo da incerteza de medição é mostrada no Quadro 1. No Quadro 2, apresentam-se os valores das resistências à compressão paralelas às fibras $\left(\mathrm{f}_{\mathrm{c} 0}\right)$ de cada corpo-de-prova, somadas às suas incertezas expandidas, considerando-se uma probabilidade de ocorrência de 95\%. Ressalta-se que foi calculado um valor de incerteza para o valor de resistência à compressão de cada CP. Logo, não existiram fontes de incerteza originadas do desvio-padrão, pois foi realizada somente uma leitura de tensão para cada corpo-de-prova. Posteriormente, os mesmos cálculos foram feitos considerando-se a média dos valores de resistência à compressão da amostra. Nesse caso, a incerteza do tipo A, devida ao desvio-padrão, foi calculada utilizando-se a equação 14 .

$$
u\left(x_{i}\right)=\frac{s\left(x_{i}\right)}{\sqrt{n}}
$$

em que:

$n$ = número de medições realizadas para a determinação de um resultado; e

$s=$ desvio-padrão relacionado a um número $\mathrm{n}$ de medições.

Quadro 1 - Cálculo da incerteza de medição do CP1

\begin{tabular}{|c|c|c|c|c|c|c|c|c|}
\hline & \multirow{2}{*}{\multicolumn{6}{|c|}{$\begin{array}{l}\text { PLANILHA PARA O CÁLCULO DE INCERTEZA - CP1 } \\
\text { ENSAIO DE COMPRESSÃO PARALELA ÀS FIBRAS }\end{array}$}} & \multirow{2}{*}{\multicolumn{2}{|c|}{\begin{tabular}{|l} 
Folha: $\quad 1 / 1$ \\
Data: \\
29/ 07/ 06 \\
\end{tabular}}} \\
\hline & & & & & & & & \\
\hline MENSURANDO: & \multicolumn{8}{|l|}{ FORÇA } \\
\hline Fontes de incerteza & valor & un & distribuição & divisor & \begin{tabular}{c|} 
coefic. de \\
sensibilidade
\end{tabular} & $\begin{array}{l}\text { incerteza } \\
\text { padrão }\end{array}$ & un & $\begin{array}{l}\text { Graus de } \\
\text { liberdade }\end{array}$ \\
\hline certificado & 0,130 & $\mathrm{kN}$ & normal & 2 & 1 & 0,065 & $\mathrm{kN}$ & infinitos \\
\hline curva de calibração & 0,017 & $\mathrm{kN}$ & normal & 1 & 1 & 0,017 & $\mathrm{kN}$ & 3 \\
\hline \multicolumn{6}{|l|}{ Incerteza combinada } & 0,067 & \multicolumn{2}{|c|}{$\mathrm{kN}$} \\
\hline MENSURANDO: & \multicolumn{8}{|c|}{ DIMENSÃO 1} \\
\hline Fontes de incerteza & valor & un & distribuição & divisor & $\begin{array}{c}\text { coeficiente de } \\
\text { sensibilidade }\end{array}$ & $\begin{array}{l}\text { incerteza } \\
\text { padrão }\end{array}$ & un & $\begin{array}{l}\text { Graus de } \\
\text { liberdade }\end{array}$ \\
\hline certificado & 0,013 & $\mathrm{~mm}$ & normal & 2 & \begin{tabular}{|l|}
1 \\
\end{tabular} & 0,007 & $\mathrm{~mm}$ & infinitos \\
\hline Resolução & 0,01 & $\mathrm{~mm}$ & triangular & 2,45 & 1 & 0,004 & $\mathrm{~mm}$ & infinitos \\
\hline \multicolumn{6}{|l|}{ Incerteza combinada } & 0,008 & \multicolumn{2}{|c|}{$\mathrm{mm}$} \\
\hline MENSURANDO: & \multicolumn{8}{|c|}{ DIMENSÄO 2} \\
\hline Fontes de incerteza & valor & un & distribuição & divisor & $\begin{array}{c}\text { coeficiente de } \\
\text { sensibilidade }\end{array}$ & $\begin{array}{l}\text { incerteza } \\
\text { padrão }\end{array}$ & un & $\begin{array}{l}\text { Graus de } \\
\text { liberdade }\end{array}$ \\
\hline certificado & 0,013 & $\mathrm{~mm}$ & normal & 2 & \begin{tabular}{|c|}
1 \\
\end{tabular} & 0,007 & $\mathrm{~mm}$ & infinitos \\
\hline Resolução & 0,01 & $\mathrm{~mm}$ & triangular & 2,45 & 1 & 0,004 & $\mathrm{~mm}$ & infinitos \\
\hline \multicolumn{6}{|l|}{ Incerteza combinada } & 0,008 & \multicolumn{2}{|c|}{$\mathrm{mm}$} \\
\hline MENSURANDO: & \multicolumn{8}{|c|}{ ÁREA = D1 X D2 } \\
\hline Fontes de incerteza & valor & un & distribuição & divisor & $\begin{array}{c}\text { coeficiente de } \\
\text { sensibilidade }\end{array}$ & $\begin{array}{l}\text { incerteza } \\
\text { padrão }\end{array}$ & un & $\begin{array}{l}\text { Graus de } \\
\text { liberdade }\end{array}$ \\
\hline Dimensão 1 & 0,008 & $\mathrm{~mm}$ & normal & 1,00 & 50,42 & 0,008 & $\mathrm{~mm}$ & infinitos \\
\hline Dimensão 2 & 0,008 & $\mathrm{~mm}$ & normal & 1,00 & 49,69 & 0,008 & $\mathrm{~mm}$ & infinitos \\
\hline \multicolumn{6}{|l|}{ Incerteza combinada } & 0,543 & \multicolumn{2}{|c|}{$\mathrm{mm} 2$} \\
\hline \multicolumn{4}{|c|}{ MENSURANDO: $\quad$ RESISTÊNCIA Ȧ COMPRESSĀO = F/A } & \multicolumn{3}{|c|}{$\begin{array}{l}\text { coef. Sensibilidade }=\delta \sigma / \delta F=1 / A \\
\text { coef. Sensibilidade }=\delta \sigma / \delta A=-F / A^{2}\end{array}$} & & \\
\hline Fontes de incerteza & valor & un & distribuição & divisor & $\begin{array}{c}\text { coeficiente de } \\
\text { sensibilidade }\end{array}$ & $\begin{array}{l}\text { incerteza } \\
\text { padrão }\end{array}$ & un & $\begin{array}{l}\text { Graus de } \\
\text { liberdade }\end{array}$ \\
\hline Força (VVC) & 0,067 & $\mathrm{kN}$ & normal & 1,00 & 0,000399143 & 0,067 & $\mathrm{kN} / \mathrm{mm}^{2}$ & 3,00 \\
\hline 2505,37 & 0,543 & $\mathrm{~mm}^{2}$ & normal & 1,00 & \begin{tabular}{|l|}
$-2,58483 \mathrm{E}-05$ \\
\end{tabular} & 0,543 & $\mathrm{kN} / \mathrm{mm}^{2}$ & inf. \\
\hline \multirow{2}{*}{\multicolumn{7}{|c|}{\begin{tabular}{|l|r|} 
Incerteza combinada & $3,026 \mathrm{E}-05$ \\
graus de liberdade efetivos &
\end{tabular}}} & \multicolumn{2}{|c|}{$\mathrm{kN} / \mathrm{mm} 2$} \\
\hline & & & & & & \multirow{2}{*}{\multicolumn{3}{|c|}{$\frac{5}{2,57}$}} \\
\hline \multicolumn{6}{|c|}{ Valor de $\mathrm{K}$ para $95 \%$ de probabilidade de ocorrência } & & & \\
\hline \multirow{2}{*}{\multicolumn{6}{|c|}{$\begin{array}{ll}\text { Incerteza expandida } \\
\text { RESULTADO DO ENSAIO }\end{array}$}} & 0,078 & \multirow{2}{*}{\multicolumn{2}{|c|}{$\frac{\mathrm{N} / \mathrm{mm}^{2}}{\mathrm{~N} / \mathrm{mm}^{2}}$}} \\
\hline & & & & & & 0,08 & & \\
\hline
\end{tabular}

Picture 1-CP1 measurement uncertainty calculation 
No Quadro 3 é mostrado o valor médio da resistência à compressão paralela às fibras $\left(f_{c 0}\right)$ da amostra considerada, somado à sua incerteza expandida, considerando-se uma probabilidade de ocorrência de 95\%.

Quadro 2 - Incertezas dos resultados dos ensaios de compressão paralela às fibras

Table 2 - Uncertainties of the compression parallel to grain test results

\begin{tabular}{|c|c|c|}
\hline $\mathrm{CP}$ & $\begin{array}{l}\text { Resultado Apresentado na } \\
\text { Máquina Universal } \mathrm{f}_{\mathrm{c} 0}(\mathrm{MPa})\end{array}$ & $\begin{array}{c}\text { Resultado (VVC) com a } \\
\text { Incerteza de Medição } \mathrm{f}_{\mathrm{c} 0}(\mathrm{MPa})\end{array}$ \\
\hline 1 & 64,650 & $64,760 \pm 0,078$ \\
\hline 2 & 63,140 & $63,240 \pm 0,077$ \\
\hline 3 & 60,170 & $60,260 \pm 0,076$ \\
\hline 4 & 60,710 & $60,810 \pm 0,075$ \\
\hline 5 & 62,540 & $62,650 \pm 0,076$ \\
\hline 6 & 60,020 & $60,120 \pm 0,075$ \\
\hline 7 & 62,190 & $62,290 \pm 0,078$ \\
\hline 8 & 60,770 & $60,870 \pm 0,076$ \\
\hline 9 & 61,960 & $62,070 \pm 0,076$ \\
\hline 10 & 63,170 & $63,270 \pm 0,077$ \\
\hline 11 & 63,220 & $63,320 \pm 0,076$ \\
\hline 12 & 61,880 & $61,980 \pm 0,076$ \\
\hline
\end{tabular}

Quadro 3 - Incertezas da média dos resultados dos ensaios de compressão paralela às fibras Table 3 - Uncertainties of the compression parallel to grain test result mean

\begin{tabular}{cc}
\hline Média dos Resultados apresentados $\mathrm{f}_{\mathrm{c} 0}(\mathrm{MPa})$ & Média dos Resultados dos VVC com a incerteza de medição $\mathrm{f}_{\mathrm{c} 0}(\mathrm{MPa})$ \\
62,04 & $62,14 \pm 1,06$
\end{tabular}

\section{CONCLUSÃO}

Os valores de incerteza obtidos dos resultados individuais da tensão de ruptura foram pouco significativos, indicando elevada qualidade dos equipamentos e boa calibração deles. Já a incerteza de medição da média da tensão de ruptura foi considerável, indicando a importância de sua consideração para a melhoria da segurança dos valores utilizados na Engenharia da Madeira.

O cálculo da incerteza é um importante parâmetro para avaliar a qualidade da medição realizada e para aumentar a confiabilidade na comparação entre valores de medições feitas por diferentes laboratórios.

\section{AGRADECIMENTOS}

A FINEP, FAPEMIG e CNPq, pela ajuda financeira na realização deste trabalho.

\section{REFERÊNCIAS}

\section{ASSOCIAÇÃO BRASILEIRA DE NORMAS} TÉCNICAS - ABNT. Projeto de estruturas de madeira - NBR 7190. Rio de Janeiro:1997. 107p.
ASSOCIAÇÃO BRASILEIRA DE NORMAS

TÉCNICAS - ABNT. Requisitos gerais para competência de laboratórios de ensaio e calibração - NBR ISO/IEC 17025. Rio de Janeiro: 2005. 20 p.

GUM - Guia para expressão da incerteza de medição. Segunda edição brasileira publicada pelo INMETRO e pela ABNT, agosto 1998.

INSTITUTO NACIONAL DE METROLOGIA, NORMALIZAÇÃO E QUALIDADE INDUSTRIAL - INMETRO. Portaria 029. Vocabulário Internacional de termos fundamentais e gerais de metrologia (VIM), 1995.

ISO/TS 14253-1. Especificações geométricas de produtos (GPS) inspeção por meio de peças e equipamentos de medição parte 1: regras decisórias para avaliar a conformidade ou a não conformidade com as especificações. Suíça. 1998. 
INSTITUTO NACIONAL DE METROLOGIA, NORMALIZAÇÃO E QUALIDADE INDUSTRIAL INMETRO. NIT-DICLA-021 - Expressão da incerteza de medição. Revisão número 02 . Rio de Janeiro: 2003. 30p.

INSTITUTO NACIONAL DE METROLOGIA, NORMALIZAÇÃO E QUALIDADE INDUSTRIAL INMETRO. NIT-DICLA-033 - Política para implementação da estimativa de incerteza de medição em laboratórios de ensaio. Ver. $n^{\circ}$ 01. Rio de Janeiro: 2005. 3p.

LOBÃO, M. S. et al. Caracterização das propriedades físico-mecânicas da madeira de eucalipto com diferentes densidades. Revista Árvore., v.28, n.6, p.889-894, 2004.

MANTILLA, E. V. C. Caracterização tecnológica da madeira de Eucalyptus cloeziana e Eucalyptus saligna cultivados em Minas Gerais. Belo Horizonte: 2006. 45p. (Relatório final FAPEMIG-EDT 1848-02)
MOREL, P.; ARRUDA, T. L.; BOHRER-MOREL, $M$. B. Cálculo de incertezas nas grandezas de influência em ensaios biológicos. In: METROSUL, 4/5 CONGRESSO LATINO-AMERICANO DE METROLOGIA, 2004, Foz do Iguaçu. A metrologia e a competitividade no mercado globalizado. Foz do Iguaçu: 2004.

OLIVEIRA, J. C. V.; SANTOS, J. A. N.

Confiabilidade e exatidão de processos de calibração: uma proposta de metodologia. In: METROSUL, 4/5 CONGRESSO LATINOAMERICANO DE METROLOGIA, 2004, Foz do Iguaçu. A metrologia e a competitividade no mercado globalizado. Foz do Iguaçu: 2004.

SERPA, P. N. et al. Avaliação de algumas propriedades da madeira de Eucalyptus grandis, Eucalyptus saligna e Pinus elliottii. Revista Árvore, v.27, n.5, p.722-733, 2003. 